\title{
Incidence and predictors of 30-day mortality in patients with ventricular septal rupture complicating acute myocardial infarction
}

\author{
Akshyaya Pradhan, ${ }^{1}$ Nirdesh Jain, ${ }^{1}$ Salvatore Cassese, ${ }^{2}$ Pravesh Vishwakarma, ${ }^{1}$ \\ Rishi Sethi, ${ }^{1}$ Sharad Chandra, ${ }^{1}$ Gaurav Chaudhary, ${ }^{1}$ Sudhanshu Kumar Dwivedi, \\ Varun Shankar Narain ${ }^{1}$
}

${ }^{1}$ Department of Cardiology, King George's Medical University, Lucknow, Uttar Pradesh, India ${ }^{2}$ Deutsches Herzzentrum München, Technische Universität München, Munchen, Germany

\section{Correspondence to} Dr Rishi Sethi, Cardiology Department, King George's Medical University, Lucknow, UP 226003, India; drrishisethi1@ gmail.com

Received 23 May 2018 Revised 17 July 2018 Accepted 19 July 2018

\section{Check for updates}

(c) Author(s) (or their employer(s)) 2018. No commercial re-use. See rights and permissions. Published by BMJ.

To cite: Pradhan A, Jain N, Cassese $\mathrm{S}$, et al. Heart Asia 2018:10:e011062. doi:10.1136/

heartasia-2018-011062

\section{ABSTRACT}

Objective We sought to investigate the incidence and predictors of 30-day mortality associated with ventricular septal rupture (VSR) complicating acute myocardial infarction (AMI) in a cohort of patients admitted to a single centre in India.

Methods From October 2013 to February 2016, a total of 6560 patients with a diagnosis of AMI were admitted to our institution. Among these patients, those with a diagnosis of VSR were retrospectively included in this registry. Clinical and echocardiographic features were collected in all cases. The primary outcome was 30-day mortality. A Cox proportional hazard regression analysis explored the predictors of 30-day mortality.

Results During the observation period, a total of 51 consecutive patients (mean age 63.8 years (9.1); $51.0 \%$ male, $41.2 \%$ were patients with diabetes) with a diagnosis of VSR complicating AMI were included. On echocardiography, left ventricular ejection fraction was $42.5 \%$ (6.5), and the most frequent location of VSR was apical (78.4\%). Overall, $27.4 \%$ of the patients received reperfusive therapy (pharmacological, 23.5\%; mechanical, $3.9 \%$ ) and $19.6 \%$ of the patients underwent surgical repair. The mean time to surgery was 7.7 days (2.4). At 30-day follow-up, death occurred in $80.4 \%$ of patients. Advanced age (HR 1.07, 95\% Cl (1.02 to 1.13), $\mathrm{p}=0.004)$, previous cerebrovascular accident (HR 52.2, $95 \% \mathrm{Cl}(3.98$ to 685.06$), \mathrm{p}=0.003)$ and surgical repair ( $\mathrm{HR} 0.05,95 \% \mathrm{Cl}(0.01$ to 0.26$), \mathrm{p}<0.001)$ were effect modifiers of the 30 -day risk of death.

Conclusions In this retrospective cohort of patients with AMI, the occurrence of VSR was not rare and carried a considerable risk of 30-day mortality. Advanced age, previous cerebrovascular accident and surgical repair influenced the risk for 30-day mortality.

\section{INTRODUCTION}

Ventricular septal rupture (VSR) is a potentially fatal complication of acute myocardial infarction (AMI). ${ }^{12}$ In the era of prompt reperfusive therapies (either pharmacological or mechanical), the incidence of VSR has considerably declined. However, the mortality among patients with AMI with VSR is reported as high as $80 \%$ even in contemporary series of patients with AMI undergoing primary percutaneous coronary intervention (PCI), and appears almost unchanged over the last few decades. ${ }^{3}$

Independent predictors associated with the development of VSR in this setting are age, female gender, previous cerebrovascular accident (CVA), impaired renal function and chronic congestive heart disease. ${ }^{4}$ At the opposite, independent predictors of survival among patients with AMI developing VSR are less studied. Surgical repair of VSR has an intuitive prognostic impact and is recommended by guideline-writing authorities regardless of haemodynamic stability at the time of diagnosis. ${ }^{5}$ However, the optimal timing of surgical repair is subject to ongoing debate. ${ }^{6}$ In addition, pending anatomical feasibility, percutaneous closure of VSR complicating AMI has attracted considerable interest as a valuable and less invasive, alternative procedure to surgery, although mortality remains high. $^{78}$

Data on VSR complicating AMI are limited in India. ${ }^{9}$ Against this background, the aim of the current report was twofold: first, to determine the incidence and 30-day mortality of VSR complicating AMI in a contemporary series of Indians admitted to a tertiary medical centre; and second, to identify predictors of 30 -day mortality.

\section{METHODS}

\section{Patient population and study protocol}

From October 2013 to February 2016, all patients with AMI admitted to the Cardiology Department, King George's Medical University, Lucknow, Uttar Pradesh, India, received an echocardiographic screening on admission and during hospital stay. Those patients with a definitive diagnosis of VSR complicating AMI (ST-segment elevation myocardial infarction) were retrospectively included in the present registry.

The presence of VSR was defined as a disruption in the ventricular septum with evidence of left-toright shunt by colour Doppler. The screening for VSR was performed via transthoracic echocardiography, while transoesophageal echocardiography was performed in case of diagnostic ambiguity or planned surgical repair. The location of the rupture at the level of the septum was defined as basal, mid or apical. The maximum distance between margins, the peak velocity and the peak gradient were measured at the level of VSR by means of a point-by-point ruler and continuous-wave Doppler measurements, as appropriate. Patients with AMI with a confirmed diagnosis of VSR at any time during hospitalisation, as well as those transferred from a referral centre, were included in this registry. Left ventricular ejection fraction (LVEF) 
6,560 eligible AMI-patients admitted from October 2013 to February 2016

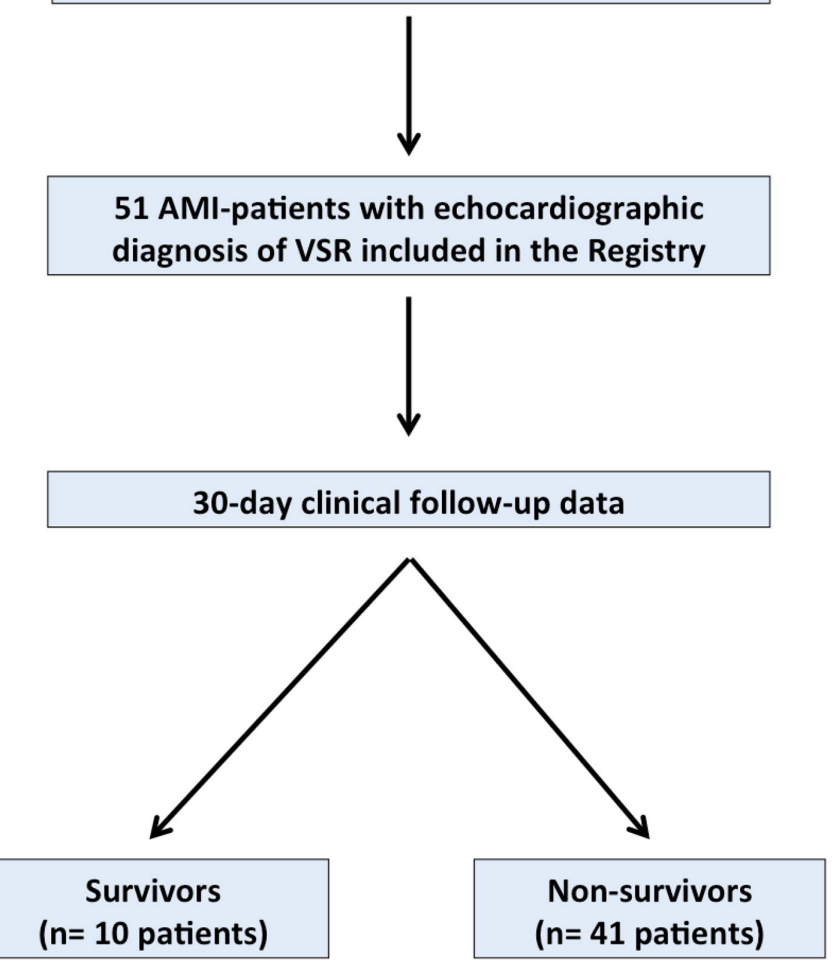

Figure 1 Flow diagram of the study. AMI, acute myocardial infarction; VSR, ventricular septal rupture.

was calculated with the biplane Simpson's method. The systolic pressure of the right ventricle (RV) was measured as a function of the peak velocity of regurgitation signal through the tricuspid valve by means of continuous-wave Doppler plus $10 \mathrm{~mm} \mathrm{Hg}$ for the right atrial pressure, assuming an intermediate to high volume overload in all cases.

In all patients, standard in-hospital therapy consisted of aspirin and thienopyridines, as clinically indicated. The use of other cardioactive drugs (including inotropic agents), as well as the choice of mechanical circulatory or ventilatory support, was left to the discretion of the treating physician. Aspirin therapy was prescribed indefinitely.

\section{Follow-up and data management}

All patients underwent follow-up by means of clinical visit at the same hospital or telephone contact. In case of an adverse event at another centre, medical records or discharge letters from other institutions were obtained and systematically reviewed. General practitioners and referring physicians were contacted for additional information, if necessary. Relevant clinical data were collected, verified against source documentation and entered into a computer database. For the current analysis, data of all patients included in the registry were transferred without patient identifiers to the ISAResearch Center (Deutsches Herzzentrum München, Technische Universität München, Munich, Germany). The final data set was checked for completeness and consistency.

\section{Endpoints definitions}

The primary endpoint of this report was all-cause death at 30-day follow-up. Death was supposed to be cardiac unless a clear non-cardiac cause could be identified. Cardiac death was defined as any death due to recurrent myocardial infarction, low-output failure, arrhythmia, complications related to in-hospital procedures and sudden cardiac death. ${ }^{10}$

\section{Statistical analysis}

Due to the small number of patients, categorical data are presented as proportions. Continuous data are presented as median (25th; 75th percentiles) and mean (SD). Baseline data were analysed in the overall cohort and expressed as unadjusted HRs with 95\% CIs at 30-day follow-up. Kaplan-Meier method served to derive the 30-day mortality rate and plot the timeto-event curve. Data for patients who did not experience the event of interest were censored at the time of the last follow-up visit. The total follow-up was defined as the time from VSR diagnosis until death, last follow-up date or 30 days, whichever came first. A Cox proportional hazard model (backward method, p value for addition to the model (0.20-0.30)) served to evaluate the extent to which patient-related characteristics influenced the 30-day probability of death. To exclude a linear association between predictors (collinearity), we estimated the variance inflation factor (a value $>10$ suggests collinearity) and inspected the correlation matrix of coefficients. The use of mechanical ventilatory support, the peak velocity of regurgitation signal through the tricuspid valve and the function of RV were not included in the final model due to collinearity. Finally, the following variables were retained in the Cox proportional hazard model for the 30-day risk of death: male gender, age (as continuous variable), diabetes mellitus, smoking habit, arterial hypertension, previous CVA, anterior AMI, AMI with RV involvement, reperfusive therapy, mechanical circulatory support, surgical repair, LVEF (as continuous variable), VSR location and increased RV systolic pressure (severe). The risk of 30-day mortality associated with these predictors was expressed as adjusted HR with 95\% CI. A formal test for model overfitting was performed calculating the Akaike's information criteria (AIC) and Schwarz's Bayesian information criteria (BIC) coefficients ${ }^{11}$ using the estat ic command. All tests were two-tailed and a $\mathrm{p}$ value $<0.05$ indicated significance. Statistical analyses were performed with Stata V.11.2 statistical software.

\section{RESULTS}

During the study period, a total of 6560 patients with a diagnosis of AMI were admitted to our institution. Among these patients, a total of $51(0.77 \%)$ had a VSR confirmed on echocardiography and entered the analyses performed for the current report. The flow diagram of the study is depicted in figure 1 .

\section{Overall population}

The baseline clinical characteristics matched a typical AMI population presenting VSR (table 1 ): the median age was 62.0 years $((60 ; 70)$, mean $63.8(9.1))$, more than half of the participants were men, roughly $40 \%$ were patients with diabetes and a fairly high proportion were active smokers. A history of AMI or CVA was present in $3.9 \%$ and $2.0 \%$ of patients, respectively. Patients presented predominantly with anterior AMI, and complained of Killip class IV on admission in the majority of cases. A slight proportion of patients presented right ventricular infarction. The median time interval between AMI and VSR diagnosis was 3 days $((1 ; 6)$, mean 4.9 (5.5)). Overall, 86.3\% of patients received mechanical ventilatory support, while few patients (3.9\%) were managed with mechanical circulatory support (intra-aortic balloon pump). Approximately a quarter of the individuals had reperfusive therapy (pharmacological or 
Table 1 Clinical features

\begin{tabular}{|c|c|c|c|c|c|}
\hline & $\begin{array}{l}\text { Overall } \\
(n=51)\end{array}$ & $\begin{array}{l}\text { HR } \\
(95 \% \mathrm{Cl}) \\
(9)_{\text {uadjust }}\end{array}$ & P values & $\begin{array}{l}\text { HR }_{\text {adjusted }} \\
(95 \% \mathrm{CI})\end{array}$ & $P$ values \\
\hline Age, years & $63.8(9.1)$ & $1.03(0.99$ to 1.07$)$ & 0.09 & $1.07(1.02$ to 1.13$)$ & 0.004 \\
\hline Male gender & 51.0 & $0.70(0.38$ to 1.30$)$ & 0.26 & 0.87 (0.39 to 1.91$)$ & 0.73 \\
\hline Diabetes mellitus & 41.2 & $0.95(0.51$ to 1.78$)$ & 0.89 & $0.57(0.25$ to 1.28$)$ & 0.17 \\
\hline Smoking habit & 25.5 & $0.76(0.37$ to 1.55$)$ & 0.45 & 1.29 (0.53 to 3.11$)$ & 0.57 \\
\hline Tobacco chewer & 23.5 & $0.95(0.45$ to 2.00$)$ & 0.89 & - & - \\
\hline Arterial hypertension & 41.2 & 1.05 (0.57 to 1.97$)$ & 0.86 & $0.76(0.35$ to 1.66$)$ & 0.50 \\
\hline Previous AMI & 3.9 & $2.50(0.58$ to 10.81$)$ & 0.22 & $3.34(0.58$ to 19.11$)$ & 0.17 \\
\hline Previous CVA & 2.0 & $12.5(1.40$ to 111.83$)$ & 0.024 & 52.23 (3.98 to 685.06$)$ & 0.003 \\
\hline Killip class on admission & & & & - & - \\
\hline III & 7.8 & $0.79(0.24$ to 2.56$)$ & 0.69 & - & - \\
\hline IV & 51.0 & $1.33(0.72$ to 2.46$)$ & 0.36 & - & - \\
\hline Anterior AMI & 74.5 & 0.77 (0.39 to 1.52$)$ & 0.46 & $1.58(0.35$ to 7.24$)$ & 0.55 \\
\hline AMI with RV involvement & 5.9 & $1.69(0.51$ to 5.85$)$ & 0.39 & $1.17(0.22$ to 6.20$)$ & 0.85 \\
\hline AMI to VSR diagnosis time, days & $4.9(5.5)$ & 0.99 (0.95 to 1.05$)$ & 0.93 & - & - \\
\hline Reperfusive therapy & 27.4 & 1.34 (0.69 to 2.61$)$ & 0.38 & $1.18(0.53$ to 2.66$)$ & 0.68 \\
\hline Lysis & 23.5 & 1.37 (0.68 to 2.75$)$ & 0.37 & - & - \\
\hline Mechanical ventilatory support & 86.3 & $6.85(1.62$ to 29.01$)$ & 0.009 & - & - \\
\hline Mechanical circulatory support & 3.9 & $2.50(0.58$ to 10.81$)$ & 0.22 & $1.70(0.34$ to 8.45$)$ & 0.51 \\
\hline Inotropic agents & 52.9 & 1.27 (0.68 to 2.37$)$ & 0.44 & - & - \\
\hline Duration of inotropic agents, days & $1.5(1.7)$ & $0.93(0.78$ to 1.09$)$ & 0.36 & - & - \\
\hline Surgical repair & 19.6 & $0.07(0.01$ to 0.31$)$ & $<0.001$ & $0.05(0.01$ to 0.26$)$ & $<0.001$ \\
\hline Time to surgery, days & $7.7(2.4)$ & $0.83(0.42$ to 1.65$)$ & 0.60 & - & - \\
\hline
\end{tabular}

Data are mean (SD) or proportion, and unadjusted/adjusted $\mathrm{HRs}$ with $95 \% \mathrm{Cl}$.

AMI, acute myocardial infarction; CVA, cerebrovascular accident; RV, right ventricle; VSR, ventricular septal rupture.

mechanical) during hospital stay. Of note, the baseline features of those patients receiving reperfusive therapy were not significantly different as compared with those of patients who did not. In two cases, VSR occurred after PCI. Of the patients, $19.6 \%$ underwent surgical repair of VSR after a median time of 7 days $((6 ; 10)$, mean $7.7(2.4))$. There were two perioperative deaths among patients undergoing surgical repair. Notably, the baseline features of those patients receiving surgical repair were not significantly different as compared with those of patients who did not.

On echocardiography (table 2), LVEF was moderately reduced. VSR was predominantly located at the apex level (78.4\%), while mid and basal locations were the less frequent septal locations. All patients with a VSR involving the apex suffered from anterior AMI, while in patients presenting with inferior AMI, VSR involved the base in all cases but two, who presented with inferior AMI and apical VSR. The mean
VSR size was 9.5 (3.8) mm, while the mean peak velocity and gradient through VSR were $3.4(0.6) \mathrm{m} / \mathrm{s}$ and 48.1 (17.6) $\mathrm{mm}$ $\mathrm{Hg}$, respectively.

\section{Survival analyses}

At 30-day follow-up, 41 patients had died (Kaplan-Meier estimate for 30-day mortality $80.4 \%$, 95\% CI (69 to 90); figure 2). Death occurred at a median of 3 days $((2 ; 4)$, mean $3.4(3.2))$ after VSR diagnosis. The baseline clinical (table 1) and echocardiographic (table 2) features were broadly similar among those who survived and those who did not. Of interest, patients who died were less likely to have received surgical repair $\left(\mathrm{HR}_{\mathrm{unad}}\right.$ justed $0.07(0.01$ to 0.31$), \mathrm{p}<0.001)$, more likely to have been treated with mechanical ventilatory support $\left(\mathrm{HR}_{\text {unadjusted }} 6.85\right.$ (1.62 to 29.01), $\mathrm{p}=0.009)$ and to have a history of CVA ( $\mathrm{HR}_{\mathrm{un}}$ adjusted 12.5 (1.40 to 111.83$), \mathrm{p}=0.024)$. Only nine patients with AMI with an apical VSR and one patient with AMI with a basal

Table 2 Echocardiographic features

\begin{tabular}{|c|c|c|c|c|c|}
\hline & Overall $(n=51)$ & $\mathrm{HR}_{\text {unadjusted }}(95 \% \mathrm{Cl})$ & $P$ values & $\mathrm{HR}_{\text {adjusted }}(95 \% \mathrm{Cl})$ & $P$ values \\
\hline LVEF & $42.5(6.5)$ & 0.98 (0.94 to 1.03$)$ & 0.58 & 0.96 (0.90 to 1.03$)$ & 0.27 \\
\hline \multicolumn{6}{|l|}{ VSR location } \\
\hline Apical & 78.4 & 0.74 (0.36 to 1.51$)$ & 0.41 & 0.34 (0.05 to 2.05$)$ & 0.24 \\
\hline VSR size, mm & $9.5(3.8)$ & 0.96 (0.88 to 1.05$)$ & 0.40 & - & - \\
\hline VSR peak velocity, $\mathrm{m} / \mathrm{s}$ & $3.4(0.6)$ & 1.17 (0.76 to 1.82 ) & 0.46 & - & - \\
\hline VSR peak gradient, mm Hg & $48.1(17.6)$ & 1.00 (0.98 to 1.01$)$ & 0.72 & - & - \\
\hline Increased RV systolic pressure & & & & - & - \\
\hline Moderate $(>35-55 \mathrm{~mm} \mathrm{Hg})$ & 47.1 & 1.14 (0.62 to 2.11$)$ & 0.66 & - & - \\
\hline Severe $(>55 \mathrm{~mm} \mathrm{Hg})$ & 17.6 & 1.09 (0.50 to 2.37$)$ & 0.82 & $1.79(0.66$ to 4.86$)$ & 0.25 \\
\hline
\end{tabular}

Data are mean (SD) or proportions, and unadjusted/adjusted $\mathrm{HRs}$ with $95 \% \mathrm{Cl}$.

LVEF, left ventricular ejection fraction; RV, right ventricle; VSR, ventricular septal rupture. 


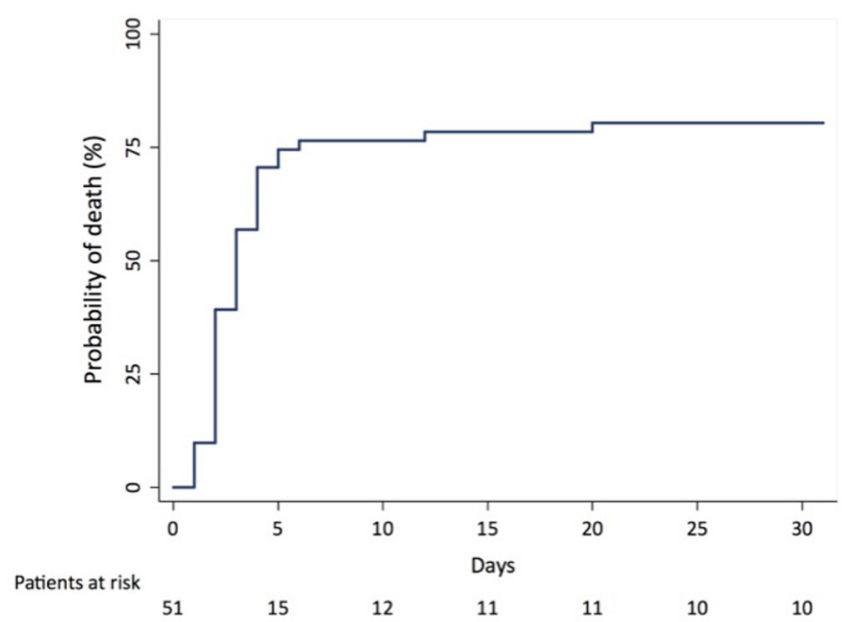

Figure 2 Survival curve at 30-day follow-up.

VSR underwent surgery. Of these, two patients (both with apical VSR) died perioperatively.

\section{Predictors of 30-day mortality}

Figure 3 displays the plots of adjusted HRs with 95\% CI of those variables displaying a significant association with 30-day risk of death. The occurrence of death was more likely in patients with advanced age $\left(\mathrm{HR}_{\text {adjusted }}\right.$ per 1-year increase, 1.07, 95\% CI (1.02 to 1.13$), \mathrm{p}=0.004)$ and previous CVA ( $\mathrm{HR}_{\text {adjusted }} 52.2,95 \% \mathrm{CI}$ (3.98 to 685.06), $\mathrm{p}=0.003$ ). Patients undergoing surgical repair of VSR showed significantly less likelihood of death $\left(\mathrm{HR}_{\text {adjusted }}\right.$ $0.05,95 \%$ CI $(0.01$ to 0.26$), \mathrm{p}<0.001)$. The values of AIC (275.5) and BIC (304.5) coefficients suggested no evidence of model overfitting.

\section{DISCUSSION}

This is a cohort of contemporary patients with AMI with a confirmed diagnosis of VSR admitted to a tertiary centre in India. The following are the main findings: (1) VSR complicating AMI is not rare in daily practice in India; (2) the 30-day mortality associated with this mechanical complication remains considerably high; and (3) advanced age, previous CVA and surgery influence the risk for 30-day mortality.

\section{Hazard ratio $[95 \% \mathrm{Cl}]$ for 30-day mortality}

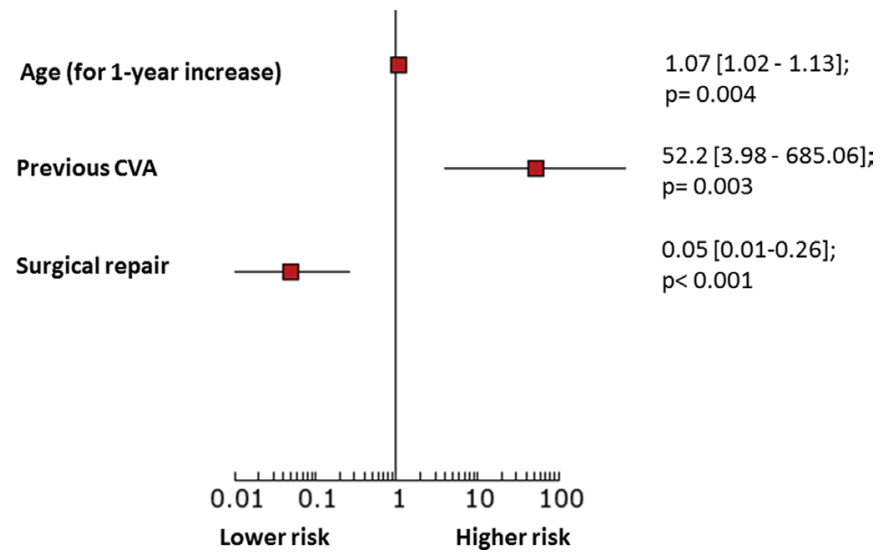

Figure 3 Significant predictors of 30-day mortality. Plots of HR for 30-day mortality according to age, previous CVA and surgical repair. The square indicates the point estimate, and the left and the right ends of the lines the $95 \% \mathrm{Cl}$. CVA, cerebrovascular accident.
This is the largest series of patients with VSR complicating AMI accumulated in India, so far. In a previous report, Harikrishnan and coworkers ${ }^{9}$ followed up 28 patients with VSR complicating AMI referred to a tertiary centre 2-3 weeks after the diagnosis. The baseline features of these individuals were broadly comparable with those of patients reported here, although they had a lower mortality risk as compared with participants of our study. This fact is attributable to a selection bias, since the previous study included only patients with AMI presenting late after VSR diagnosis. In addition, Harikrishnan and coworkers ${ }^{9}$ did not investigate the predictors of mortality, as we did.

While interpreting the study outcomes, certain points are noteworthy to remember. First, modern reperfusive strategies and more potent antiplatelet therapies have modified the epidemiology of VSR complicating $\mathrm{AMI}^{3}$ : in fact, the incidence of this complication has dramatically declined and is reported as high as $0.2 \%$ in recent primary PCI studies. ${ }^{12}$ In the current series, we found a slightly higher incidence of VSR complicating AMI $(0.77 \%)$. This registry includes participants admitted to a tertiary medical centre, where patients have high disease severity. In addition, some patients with AMI were referred to our centre due to the diagnosis of VSR, a fact that could have inflated the observed incidence of this mechanical complication.

Second, necropsy studies of patients experiencing fatal AMI have consistently demonstrated that VSR occurs as a consequence of a transmural infarction. ${ }^{13}$ In this respect, the reduction of ischaemia time among patients with AMI pursued in the last few decades in Western countries has generally reduced the incidence and shortened the time to diagnosis of VSR complicating AMI. ${ }^{12}$ Indeed, recent data demonstrate that VSR complicating AMI is diagnosed earlier (within 24 hours) as compared with historical cohorts (3-5 days). ${ }^{3}$ In the current report, $27.4 \%$ of the patients received reperfusive therapy, and the median time interval between AMI and VSR diagnosis was 3 days. There were no baseline differences between patients undergoing reperfusion and those who did not. The efforts in reducing delays, simplifying access to hospitals and providing affordable treatments for patients with AMI should represent the main objectives of care practitioners in India, in order to lower the burden of morbidity and mortality associated with cardiovascular disease in this geographical area. ${ }^{14}$ Notwithstanding this, in patients with AMI developing VSR, prompt mechanical reperfusion does not reduce 30-day mortality. ${ }^{15}$ Consistently, in our analysis, we did not find a survival benefit associated with reperfusive therapy $\left(\mathrm{HR}_{\text {adjusted }} 1.18,95 \% \mathrm{CI}(0.53\right.$ to 2.66$\left.), \mathrm{p}=0.68\right)$.

Finally, we found that advanced age, previous CVA and surgical repair were effect modifiers of 30-day mortality risk in patients with AMI developing VSR. Advanced age and previous CVA may underline a higher comorbidity, which likely explains the statistical association of these variables with higher risk of death at 30 days observed in this report. Although a surgical repair is highly recommended for patients with AMI developing VSR regardless of the haemodynamic status, the timing of the surgical repair remains under consideration. In the current series, patients underwent surgery after a median time of 7 days and displayed 30 -day mortality as high as $20 \%$. Previous data show that in patients with AMI developing VSR, individuals undergoing surgical repair within 24 hours have the highest mortality $(>60 \%)$, with a dramatic drop-off in those cases receiving surgery $>7$ days. ${ }^{616}$ In our registry, the timing at which patients underwent surgical repair may explain the reported favourable outcome, since a delayed surgery may have improved stability of the cardiac tissue and allowed a more effective repair of the VSR. In contrast, early surgery is usually performed on 
individuals with haemodynamic instability; thus, the improved outcome with delayed surgery may represent a selection bias. ${ }^{17}$

\section{Study limitations}

This study presents some limitations. First, we studied a retrospective cohort, with the inherent limitations of this kind of analyses. Second, the diagnosis of VSR in patients with AMI was echocardiography-based and the possibility for missed diagnoses should be considered. Third, this limited population of patients with AMI with a diagnosis of VSR has inadequate statistical power and the number of statistically significant variables among groups was scarce. In this respect, we cannot ensure that all potential confounders have been considered. Fourth, among the entire cohort of patients with AMI admitted to our institution during the study period, data on the type of myocardial infarction (with or without ST-segment elevation) and the time from symptom onset to reperfusive therapy in individuals with VSR complicating AMI were not routinely collected. Fifth, clinical decisions on treatment were not controlled: a biased treatment allocation cannot be completely excluded, although we did not record baseline differences among patients allocated to reperfusive or surgical therapies. Sixth, the techniques used for surgical repair were not systematically recorded in this study and a possible survival benefit associated with certain surgical (or percutaneous) techniques cannot be investigated in this context. Finally, all patients were enrolled at a single centre in India: ethnicity plays an important prognostic role and people belonging to South Asian countries are among those with the greater mortality and morbidity from coronary heart disease as compared with Western countries. ${ }^{18}$ In this respect, current findings do not apply to other institutions or geographical areas.

\section{CONCLUSIONS}

In this retrospective cohort of patients with AMI admitted to a single tertiary centre in India, the occurrence of VSR was

\section{Key messages}

\section{What is already known about this subject?}

- Ventricular septal rupture (VSR) complicating acute myocardial infarction carries high mortality even in the reperfusion era.

- Data on VSR in acute myocardial infarction from India are limited.

\section{What does this study add?}

- This study is the largest series of VSR complicating acute myocardial infarction from the Indian subcontinent.

- The 30-day mortality of such patients was very high (80.4\%).

- Only a quarter (27.4\%) of these patients had received any form of reperfusion therapy.

- Advanced age, prior cerebrovascular events and surgical repair were found to be independent modifiers of 30-day mortality.

\section{How might this impact on clinical practice?}

- VSR continues to impose high mortality in patients with STsegment elevation myocardial infarction (STEMI).

- Surgical correction may lead to improved survival despite high perioperative mortality.

- All attempts should be directed for timely reperfusion of patients with STEMI. not rare and associated with considerable 30-day mortality. Advanced age, previous CVA and surgical repair influenced the risk for 30-day mortality.

Contributors All the authors played their part in the build-up of this collaborative research project. AP conceived the idea and NJ prepared the draft protocol. Subsequently, PV and GC pitched in for data collection, and the process was completed over years of collective effort. When the datasheet was ready, SCa and RS began data analysis with PV and AP. After the completion of data interpretation, GC was roped in again for drafting the article. Then it was upon the trio of VSN, SKD and SCh to critically review the article and send back to RS. RS initiated the final discussions with NJ, SCa and AP to come out with the final version of the article. The first version was submitted by $\mathrm{NJ}$, but since he had relocated the revised version was submitted by RS in consultation with SCa and AP. SCa was pivotal in revising the article and giving point-by-point rebuttal. AP reviewed the revised version and submitted back the same online. The second revision was done by SCa and AP, guided by RS. Final revision was completed by SCa, while AP reviewed and submitted it online. Conception or design of the work: AP, NJ. Data collection: NJ, AP, PV, GC. Data analysis and interpretation: SCa, RS, PV, AP. Drafting the article: SCa, RS, AP, GC Critical revision of the article: VSN, SKD, SCh, RS. Final approval of the version to be published: SCa, NJ, AP, RS. Manuscript submission and revision: SCa, RS, AP. Second revision: SCa, AP, RS. Third revision: SCa, AP.

Funding The authors have not declared a specific grant for this research from any funding agency in the public, commercial or not-for-profit sectors.

Competing interests None declared.

Patient consent Not required.

Provenance and peer review Not commissioned; internally peer reviewed.

\section{REFERENCES}

1. Birnbaum Y, Fishbein MC, Blanche $C$, et al. Ventricular septal rupture after acute myocardial infarction. N Engl J Med 2002;347:1426-32.

2. López-Sendón J, Gurfinkel EP, Lopez de Sa E, et al. Factors related to heart rupture in acute coronary syndromes in the Global Registry of Acute Coronary Events. Eur Heart J 2010;31:1449-56.

3. Jones BM, Kapadia SR, Smedira NG, et al. Ventricular septal rupture complicating acute myocardial infarction: a contemporary review. Eur Heart J 2014;35:2060-8.

4. Menon V, Webb JG, Hillis LD, et al. Outcome and profile of ventricular septal rupture with cardiogenic shock after myocardial infarction: a report from the SHOCK Trial Registry. SHould we emergently revascularize Occluded Coronaries in cardiogenic shock? J Am Coll Cardiol 2000;36(Suppl A):1110-6.

5. American College of Emergency Physicians, Society for Cardiovascular Angiography and Interventions, O'Gara PTet al. 2013 ACCF/AHA guideline for the management of ST-elevation myocardial infarction: a report of the American college of cardiology foundation/American heart association task force on practice guidelines. J Am Coll Cardiol 2013;61:e78-140

6. Papalexopoulou N, Young CP, Attia RQ. What is the best timing of surgery in patients with post-infarct ventricular septal rupture? Interact Cardiovasc Thorac Surg 2013;16:193-6.

7. Thiele $\mathrm{H}$, Kaulfersch $\mathrm{C}$, Daehnert I, et al. Immediate primary transcatheter closure of postinfarction ventricular septal defects. Eur Heart J 2009;30:81-8.

8. Assenza GE, McElhinney DB, Valente AM, et al. Transcatheter closure of postmyocardial infarction ventricular septal rupture. Circ Cardiovasc Interv 2013;6:59-67.

9. Harikrishnan S, Tharakan J, Titus T, et al. Ventricular septal rupture following myocardial infarction: clinical, haemodynamic, angiographic profile and long-term outcome. Int J Cardiol 2007;120:279-80.

10. Cutlip DE, Windecker $S$, Mehran R, et al. Clinical end points in coronary stent trials: a case for standardized definitions. Circulation 2007;115:2344-51.

11. Schwarz G. Estimating the dimension of a model. The Annals of Statistics 1978;6:461-4.

12. APEX AMI Investigators, Armstrong PW, Granger CB, et al. Pexelizumab for acute STelevation myocardial infarction in patients undergoing primary percutaneous coronary intervention: a randomized controlled trial. JAMA 2007:297:43-51.

13. Roberts WC, Burks KH, Ko JM, et al. Commonalities of cardiac rupture (left ventricular free wall or ventricular septum or papillary muscle) during acute myocardial infarction secondary to atherosclerotic coronary artery disease. Am J Cardiol 2015;115:125-40.

14. Xavier D, Pais P, Devereaux PJ, et al. Treatment and outcomes of acute coronary syndromes in India (CREATE): a prospective analysis of registry data. Lancet 2008;371:1435-42.

15. French JK, Hellkamp AS, Armstrong PW, et al. Mechanical complications after percutaneous coronary intervention in ST-elevation myocardial infarction (from APEXAMI). Am J Cardiol 2010;105:59-63. 


\section{Original research}

16. Sharma YP, Kamana NK, Vadivelu R. Precision in cardiology: should all cases of myocardial infarction with ventricular septal rupture require early repair? Heart Asia 2013;5:235-7.
17. Liebelt JJ, Yang Y, DeRose JJ, et al. Ventricular septal rupture complicating acute myocardial infarction in the modern era with mechanical circulatory support: a single center observational study. Am J Cardiovasc Dis 2016;6:10-16.

18. Ueshima H, Sekikawa A, Miura K, et al. Cardiovascular disease and risk factors in Asia: a selected review. Circulation 2008;118:2702-9. 